\title{
A NUMERICAL METHOD FOR A SECOND ORDER SINGULARLY PERTURBED FREDHOLM INTEGRO-DIFFERENTIAL EQUATION
}

\author{
GABIL M. AMIRALIYEV, MUHAMMET ENES DURMAZ, AND MUSTAFA KUDU
}

Received 8 April, 2019

\begin{abstract}
The boundary-value problem for a second order singularly perturbed Fredholm integrodifferential equation was considered in this paper. For the numerical solution of this problem, we use an exponentially fitted difference scheme on a uniform mesh which is succeeded by the method of integral identities with the use of exponential basis functions and interpolating quadrature rules with the weight and remainder terms in integral form. Also, the method is first order convergent in the discrete maximum norm. Numerical example shows that recommended method has a good approximation characteristic.
\end{abstract}

2010 Mathematics Subject Classification: 65L11; 65L12; 65L20; 65R20

Keywords: Fredholm integro-differential equation, singular perturbation, finite difference, uniform convergence

\section{INTRODUCTION}

Fredholm integro-differential equations (FIDEs) have in large quantities applications in every branches of science. FIDEs arise from the mathematical modeling of many scientific phenomena, such as the study of fluid, physics, chemistry, biology, mechanics, astronomy, potential theory, electrostatics, control theory of industrial mathematics and chemical kinetics $[13,14,18]$. On the other hand, FIDEs are quite difficult to find exact solutions. For this reason, numerical methods play a significant role in this problems, for example, in [5, 8-11](see, also references therein).

Below, the boundary-value problem for a singularly perturbed Fredholm integrodifferential equation(SPFIDE) is considered:

$$
\begin{aligned}
& L u:=-\varepsilon u^{\prime \prime}+a(x) u+\lambda \int_{0}^{l} K(x, s) u(s) d s=f(x), \quad x \in(0, l), \\
& u(0)=A, \quad u(l)=B,
\end{aligned}
$$

where $\varepsilon \in(0,1]$ is a perturbation parameter, $\lambda$ is real parameter. We assume that $a(x) \geq \alpha>0, f(x)$ and $K(x, s)$ are the sufficiently smooth functions satisfying certain 
regularity conditions to be specified. The solution $u(x)$ of (1.1) has in general a boundary layer near $x=0$ and $x=l$.

Singularly perturbed differential equations are typically characterized by a small parameter $\varepsilon$ multiplying some or all of the highest order terms in the differential equation. This problem undergo rapid changes within very thin layers near the boundary or inside the problem domain, so most of the conventional methods fail when this small parameter approaches to zero. These singularly perturbed differential equations arise in the modeling of various modern complicated processes, such as reactiondiffusion processes, epidemic dynamics, high Reynold's number flow in the fluid dynamics, heat transport problem. For more details on singular perturbation, one can refer the books $[4,15-17,19]$ and the references therein. Survey of some existence and uniqueness results of singularly perturbed equations can be found in [7,15-17].

In recent years, there has been a growing interest in the numerical solution of integral equations. The Adomian decomposition method for solving linear second-order FIDEs is presented in [10]. Qing Xue et al. [20] studied on an improved reproducing kernel method to find the numerical solution of FIDE type boundary value problems. Emamzadeh and Kajani [6] used a numerical method for solving the nonlinear Fredholm integral equation. Jackiewicz et al. [9] proposed several approaches to the numerical solution of a new FIDEs modelling neural networks. Gegele et al. [8] presented some approximation methods to solve higher order linear FIDEs. Karimi and Jozi [11] proposed a new numerical method for solving system of linear Fredholm integral equations of the second kind.

The above mentioned papers, related to FIDEs were concerned only with the regular cases. Also, current studies for the numerical solution of SPFIDEs have not widespread yet. Various difference schemes for singularly perturbed integro-differential equations and problems with integral boundary condition were investigated in [3,12].

In this paper, we present fitted type difference scheme on an uniform mesh for the numerical solution of the problem (1.1). The difference scheme is constructed by the method of integral identities with the use exponential basis functions and interpolating quadrature rules with the weight and remainder terms in integral form [1]. To approximate the integral part of (1.1), the composite right-side rectangle rule with the remainder term in integral form is being used.

The organization of the paper is as follows. In Section 2, we state some significant properties of the exact solution. In Section 3, we describe the finite difference discretization and appropriate mesh. The error analysis for the approximate solution is presented in Section 4. Uniform convergence is proved in the discrete maximum norm. Numerical results are given in Section 5 to support the predicted theory. The paper ends with a summary of the main conclusions.

Notation 1. Throughout the paper, $C$ will denote a generic positive constant independent of $\varepsilon$ and the mesh parameter and $\|g\|_{\infty}$ is the continuous maximum norm on the corresponding closed interval for any continuous function $g(x)$. 


\section{The Continuous Problem}

Lemma 1. If $a, f \in C^{1}[0, l]$, $\frac{\partial^{s} K}{\partial x^{s}} \in C[0, l]^{2},(s=0,1)$ and

$$
|\lambda|<\frac{\alpha}{\max _{0 \leq x \leq l} \int_{0}^{l}|K(x, s)| d s},
$$

then for the solution $u(x)$ of the problem (1.1) hold the following estimates

$$
\begin{aligned}
\|u\|_{\infty} & \leq C, \\
\left|u^{\prime}(x)\right| & \leq C\left\{1+\frac{1}{\sqrt{\varepsilon}}\left(e^{-\frac{\sqrt{\alpha} x}{\sqrt{\varepsilon}}}+e^{-\frac{\sqrt{\alpha}(l-x)}{\sqrt{\varepsilon}}}\right)\right\}, \quad x \in[0, l] .
\end{aligned}
$$

Proof. Using the maximum principle for the operator $L_{0} u=-\varepsilon u^{\prime \prime}+a(x) u$, we obtain the estimate

$$
\|u\|_{\infty} \leq|A|+|B|+\alpha^{-1}|| f \|_{\infty}+\alpha^{-1}|\lambda| \max _{0 \leq x \leq l} \int_{0}^{l}|K(x, s)||u(s)| d s
$$

which after taking into account (2.1), leads to (2.2).

Next, we prove the estimate (2.3). Using (2.2) on (1.1) we have

$$
\left|u^{\prime \prime}(x)\right|=\frac{1}{\varepsilon}\left|f(x)-a(x) u(x)-\lambda \int_{0}^{l} K(x, s) u(s) d s\right| \leq \frac{C}{\varepsilon}, \quad 0 \leq x \leq l .
$$

Moreover, we now proceed with the estimation of $\left|u^{\prime}(0)\right|,\left|u^{\prime}(l)\right|$. Here we use the following relation which holds for any function $g \in C^{2}[0, l]$ :

$$
g^{\prime}(x)=g\left[\alpha_{0}, \alpha_{1}\right]-\int_{\alpha_{0}}^{\alpha_{1}} K_{0}(\xi, x) g^{\prime \prime}(\xi) d \xi, \quad \alpha_{0}<\alpha_{1},
$$

where

$$
\begin{aligned}
g\left(\alpha_{0} ; \alpha_{1}\right) & =\frac{g\left(\alpha_{1}\right)-g\left(\alpha_{0}\right)}{\alpha_{1}-\alpha_{0}} \\
K_{0}(\xi, x) & =T_{0}(\xi-x)-\left(\alpha_{1}-\alpha_{0}\right)^{-1}\left(\xi-\alpha_{0}\right)
\end{aligned}
$$

and

$$
T_{0}(\lambda)= \begin{cases}1, & \lambda \geq 0 \\ 0, & \lambda<0\end{cases}
$$


Equality (2.4) with the values $g(x)=u(x), x=0, \alpha_{0}=0$, and $\alpha_{1}=\sqrt{\varepsilon}$ yields

$$
\left|u^{\prime}(0)\right| \leq \frac{u(\sqrt{\varepsilon})-u(0)}{\sqrt{\varepsilon}}-\int_{0}^{\sqrt{\varepsilon}} K_{0}(\xi, 0) u^{\prime \prime}(\xi) d \xi \leq \frac{C}{\sqrt{\varepsilon}} .
$$

Similarly, using (2.4) for $g(x)=u(x), x=l, \alpha_{0}=l-\sqrt{\varepsilon}$, and $\alpha_{1}=l$ we confirm that

$$
\left|u^{\prime}(l)\right| \leq \frac{u(l)-u(\sqrt{\varepsilon})}{\sqrt{\varepsilon}}-\int_{l-\sqrt{\varepsilon}}^{l} K_{0}(\xi, l) u^{\prime \prime}(\xi) d \xi \leq \frac{C}{\sqrt{\varepsilon}} .
$$

Next, differentiating (1.1), according to (2.5) and (2.6), we get

$$
-\varepsilon v^{\prime \prime}+a(x) v=F(x), \quad v(0)=O\left(\frac{1}{\sqrt{\varepsilon}}\right), \quad v(l)=O\left(\frac{1}{\sqrt{\varepsilon}}\right)
$$

with

$$
v(x)=u^{\prime}(x), \quad F(x)=f^{\prime}(x)-a^{\prime}(x) u(x)-\lambda \int_{0}^{l} \frac{\partial}{\partial x} K(x, s) u(s) d s .
$$

By virtue of (2.2) evidently

$$
|F(x)| \leq C .
$$

In order to estimate the solution of the problem (2.7), we present it in the form

$$
v(x)=v_{1}(x)+v_{2}(x),
$$

where the functions $v_{1}(x)$ and $v_{2}(x)$ are the solutions of the following problems respectively:

$$
\begin{gathered}
-\varepsilon v_{1}^{\prime \prime}+a(x) v_{1}=F(x), \\
v_{1}(0)=v_{1}(l)=0, \\
-\varepsilon v_{2}^{\prime \prime}+a(x) v_{2}=0, \\
v_{2}(0)=O\left(\frac{1}{\sqrt{\varepsilon}}\right), \quad v_{2}(l)=O\left(\frac{1}{\sqrt{\varepsilon}}\right) .
\end{gathered}
$$

For the solution of the problem (2.9), using the maximum principle and (2.8), we have

$$
\left|v_{1}(x)\right| \leq \alpha^{-1}|| F \|_{\infty} \leq C, \quad 0 \leq x \leq l .
$$

According to the maximum principle, from the problem (2.10), we also conclude that

$$
\left|v_{2}(x)\right| \leq w(x),
$$


where the function $w(x)$ is the solution of the following problem:

$$
\begin{gathered}
-\varepsilon w^{\prime \prime}+\alpha w=0, \\
w(0)=\left|v_{2}(0)\right|, \quad w(l)=\left|v_{2}(l)\right| .
\end{gathered}
$$

The solution of problem (2.13) is given by

$$
w(x)=\frac{1}{\sinh \left(\frac{\sqrt{\alpha} l}{\sqrt{\varepsilon}}\right)}\left\{w(0) \sinh \left(\frac{\sqrt{\alpha}(l-x)}{\sqrt{\varepsilon}}\right)+w(l) \sinh \left(\frac{\sqrt{\alpha} x}{\sqrt{\varepsilon}}\right)\right\} .
$$

Hence, taking into consideration (2.10) we obtain

$$
w(x) \leq \frac{C}{\sqrt{\varepsilon}}\left\{e^{-\frac{\sqrt{\alpha} x}{\sqrt{\varepsilon}}}+e^{-\frac{\sqrt{\alpha}(l-x)}{\sqrt{\varepsilon}}}\right\} .
$$

Finally, the use bounds (2.11), (2.12) and (2.14) in the inequality

$$
\left|u^{\prime}(x)\right| \leq\left|v_{1}(x)\right|+\left|v_{2}(x)\right|
$$

immediately leads to (2.3).

\section{The Mesh And Discretization}

Let $\omega_{N}$ be an uniform mesh on $[0, l]$ :

$$
\omega_{N}=\left\{x_{i}=i h, i=1,2, \ldots, N-1, h=\frac{l}{N}\right\}
$$

and

$$
\bar{\omega}_{N}=\omega_{N} \cup\left\{x=0, x_{N}=l\right\} .
$$

To construct the difference scheme for the problem (1.1), we start with the following identity

$$
\chi_{i}^{-1} h^{-1} \int_{x_{i-1}}^{x_{i+1}} L u(x) \varphi_{i}(x) d x=\chi_{i}^{-1} h^{-1} \int_{x_{i-1}}^{x_{i+1}} f(x) \varphi_{i}(x) d x, i=1,2, . ., N-1,
$$

with the basis functions

$$
\varphi(x)= \begin{cases}\varphi_{i}^{(1)}(x) \equiv \frac{\sinh \gamma_{i}\left(x-x_{i}\right)}{\sinh \gamma_{i} h}, & x_{i-1}<x<x_{i}, \\ \varphi_{i}^{(2)}(x) \equiv \frac{\sinh \gamma_{i}\left(x_{i+1}-x\right)}{\sinh \gamma_{i} h}, & x_{i}<x<x_{i+1}, \\ 0, & x \notin\left(x_{i-1}, x_{i+1}\right),\end{cases}
$$

where

$$
\gamma_{i}=\sqrt{\frac{a\left(x_{i}\right)}{\varepsilon}}, \quad \chi_{i}=h^{-1} \int_{x_{i-1}}^{x_{i+1}} \varphi_{i}(x) d x=\frac{2 \tanh \left(\gamma_{i} h / 2\right)}{\gamma_{i} h} .
$$


We note that the functions $\varphi_{i}^{(1)}$ and $\varphi_{i}^{(2)}$ are the solutions of the following problems respectively:

$$
\begin{array}{rlrl}
-\varepsilon \varphi^{\prime \prime}+a_{i}(x) \varphi(x) & =0, & x_{i-1}<x<x_{i} \\
\varphi\left(x_{i-1}\right) & =0, & \varphi\left(x_{i}\right) & =1, \\
-\varepsilon \varphi^{\prime \prime}+a_{i}(x) \varphi(x) & =0, & x_{i}<x<x_{i+1} \\
\varphi\left(x_{i}\right) & =1, & \varphi\left(x_{i+1}\right) & =0 .
\end{array}
$$

By using the method of exact difference schemes (see e.g. [1-3]), it follows that

$$
\begin{aligned}
& -\chi_{i}^{-1} h^{-1} \varepsilon \int_{x_{i-1}}^{x_{i+1}} \varphi_{i}(x) u^{\prime \prime}(x) d x+\chi_{i}^{-1} h^{-1} a_{i} \int_{x_{i-1}}^{x_{i+1}} \varphi_{i}(x) u(x) d x= \\
& -\varepsilon \chi_{i}^{-1}\left\{1+a_{i} \varepsilon^{-1} \int_{x_{i-1}}^{x_{i}} \varphi_{i}^{(1)}(x)\left(x-x_{i}\right) d x\right\} u_{\bar{x} x, i} \\
& +a_{i} \chi_{i}^{-1}\left\{h^{-1} \int_{x_{i-1}}^{x_{i}} \varphi_{i}^{(1)} d x+h^{-1} \int_{x_{i}}^{x_{i+1}} \varphi_{i}^{(2)} d x\right\} u_{i}=-\varepsilon \theta_{i} u_{\bar{x} x, i}+a_{i} u_{i}
\end{aligned}
$$

with

$$
\theta_{i}=\frac{a_{i} \rho^{2}}{4 \sinh ^{2}\left(\sqrt{a_{i}} \rho / 2\right)}, \quad \rho=\frac{h}{\sqrt{\varepsilon}}
$$

Thereby

$$
\chi_{i}^{-1} h^{-1} \int_{x_{i-1}}^{x_{i+1}}\left[\varepsilon u^{\prime \prime}(x)+a(x) u(x)\right] \varphi_{i}(x) d x=-\varepsilon \theta_{i} u_{\bar{x} x, i}+a_{i} u_{i}+R_{i}^{(1)}
$$

with remainder term

$$
R_{i}^{(1)}=\chi_{i}^{-1} h^{-1} \int_{x_{i-1}}^{x_{i+1}}\left[a(x)-a\left(x_{i}\right)\right] u(x) \varphi_{i}(x) d x .
$$

Further for the right-side in (3.1) we have

$$
\chi_{i}^{-1} h^{-1} \int_{x_{i-1}}^{x_{i+1}} f(x) \varphi_{i}(x) d x=f_{i}+R_{i}^{(2)}
$$

with remainder term

$$
R_{i}^{(2)}=\chi_{i}^{-1} h^{-1} \int_{x_{i-1}}^{x_{i+1}}\left[f(x)-f\left(x_{i}\right)\right] \varphi_{i}(x) d x
$$


For integral term involving kernel function, we have from (3.1)

$$
\begin{aligned}
\chi_{i}^{-1} h^{-1} \int_{x_{i-1}}^{x_{i+1}} d x \varphi_{i}(x) \int_{0}^{l} K(x, s) u(s) d s & =\int_{0}^{l} K\left(x_{i}, s\right) u(s) d s \\
& +\chi_{i}^{-1} h^{-1} \int_{x_{i-1}}^{x_{i+1}} d x \varphi_{i}(x) \int_{0}^{l}\left[K(x, s)-K\left(x_{i}, s\right)\right] u(s) d s .
\end{aligned}
$$

Further using the composite right side rectangle rule, we obtain

$$
\left.\int_{0}^{l} K\left(x_{i}, s\right) u(s) d s=h \sum_{j=1}^{N} K_{i j} u_{j}-\sum_{j=1}^{N} \int_{x_{j-1}}^{x_{j}}\left(\xi-x_{j-1}\right) \frac{\partial}{\partial \xi}\left[K\left(x_{i}, \xi\right) u(\xi)\right)\right] d \xi .
$$

Therefore we get

$$
\chi_{i}^{-1} h^{-1} \int_{x_{i-1}}^{x_{i+1}} d x \varphi_{i}(x) \int_{0}^{l} K(x, s) u(s) d s=h \sum_{j=1}^{N} K_{i j} u_{j}+R_{i}^{(3)}
$$

with remainder term

$$
\begin{aligned}
R_{i}^{(3)}= & \chi_{i}^{-1} h^{-1} \lambda \int_{x_{i-1}}^{x_{i+1}} d x \varphi_{i}(x) \int_{0}^{l}\left[K(x, s)-K\left(x_{i}, s\right)\right] u(s) d s \\
& \left.-\lambda \sum_{j=1}^{N} \int_{x_{j-1}}^{x_{j}}\left(\xi-x_{j-1}\right) \frac{\partial}{\partial \xi}\left[K\left(x_{i}, \xi\right) u(\xi)\right)\right] d \xi .
\end{aligned}
$$

The relations (3.2), (3.4) and (3.6) yield the following exact relation for $u\left(x_{i}\right)$

$$
L_{N} u_{i}:=-\varepsilon \theta_{i} u_{\bar{x} x, i}+a_{i} u_{i}+\lambda h \sum_{j=1}^{N} K_{i j} u_{j}+R_{i}=f_{i}, \quad 1 \leq i \leq N-1
$$

with remainder term

$$
R_{i}=R_{i}^{(1)}+R_{i}^{(2)}+R_{i}^{(3)},
$$

where $R_{i}^{(k)} ;(k=1,2,3)$ are defined by (3.3), (3.5) and (3.7) respectively. Based on (3.8) we propose the following difference scheme for approximating (1.1).

$$
\begin{aligned}
L_{N} y_{i} & :=-\varepsilon \theta_{i} y_{\bar{x} x, i}+a_{i} y_{i}+\lambda h \sum_{j=1}^{N} K_{i j} y_{j}=f_{i}, \quad 1 \leq i \leq N-1, \\
y_{0} & =A, \quad y_{N}=B .
\end{aligned}
$$




\section{ERROR ANALYSIS}

From (3.8) and (3.10) for the error of the approximate solution $z_{i}=y_{i}-u_{i}$ we have

$$
\begin{aligned}
L_{N} z_{i} & :=-\varepsilon \theta_{i} z_{\bar{x} x, i}+a_{i} z_{i}+\lambda h \sum_{j=1}^{N} K_{i j} z_{j}=R_{i}, \quad 1 \leq i \leq N-1, \\
z_{0} & =0, \quad z_{N}=0 .
\end{aligned}
$$

where $R_{i}$ are defined by (3.9).

Theorem 1. Under the conditions of Lemma (2.1) and

$$
|\lambda|<\frac{\alpha}{\max _{1 \leq i \leq N} \sum_{j=1}^{N} h\left|K_{i j}\right|},
$$

the solution of (3.10) converges $\varepsilon$-uniformly to the solution of (1.1). For the error of approximate solution the following estimate hols

$$
\|y-u\|_{\infty, \bar{\omega}_{N}} \leq C h
$$

Proof. Applying the maximum principle, from (4.1) we have

$$
\begin{aligned}
\|z\|_{\infty, \bar{\omega}_{N}} \leq \alpha^{-1}|| R-\lambda h \sum_{j=1}^{N} K_{i j} z_{j} \|_{\infty, \omega_{N}} & \\
& \leq \alpha^{-1}\|R\|_{\infty, \omega_{N}}+|\lambda| \alpha^{-1} \max _{1 \leq i \leq N} \sum_{j=1}^{N} h\left|K_{i j}\right|\|z\|_{\infty, \bar{\omega}_{N}}
\end{aligned}
$$

hence

$$
\|z\|_{\infty, \bar{\omega}_{N}} \leq \frac{\alpha^{-1}|| R \|_{\infty, \omega_{N}}}{1-|\lambda| \alpha^{-1} \max _{1 \leq i \leq N} \sum_{j=1}^{N} h\left|K_{i j}\right|}
$$

which implies of

$$
\|z\|_{\infty, \bar{\omega}_{N}} \leq C|| R \|_{\infty, \omega_{N}}
$$

Further we estimate $R_{i}^{(1)}, R_{i}^{(2)}$ and $R_{i}^{(3)}$ seperately. For $a(x)$, by the mean value theorem, we have

$$
\left|a(x)-a\left(x_{i}\right)\right| \leq\left|a^{\prime}\left(\xi_{i}\right)\right|\left|x-x_{i}\right| \leq C h, \quad x_{i} \leq \xi_{i} \leq x .
$$


Thereby for $R_{i}^{(1)}$, by $a \in C^{1}[0, l]$ and (2.2) we get

$$
\begin{aligned}
\left|R_{i}^{(1)}\right| & \leq \chi_{i}^{-1} h^{-1}\left|\int_{x_{i-1}}^{x_{i+1}}\left[a(x)-a\left(x_{i}\right)\right] u(x) \varphi_{i}(x) d x\right| \\
& \leq C h \chi_{i}^{-1} h^{-1} \int_{x_{i-1}}^{x_{i+1}} \varphi_{i}(x) d x=C h .
\end{aligned}
$$

Similarly, for $R_{i}^{(2)}$ we get

$$
\left|R_{i}^{(2)}\right| \leq C h
$$

Finally for $R_{i}^{(3)}$, taking into account the boundedness of $\frac{\partial K}{\partial x}$ and (2.3) it follows that

$$
\begin{aligned}
\left|R_{i}^{(3) \mid \leq} \leq \chi_{i}^{-1} h^{-1}\right| \lambda\left|\int_{x_{i-1}}^{x_{i+1}} d x \varphi_{i}(x) \int_{0}^{l}\right| K\left(x_{i}, s\right)-K(x, s)|| u(s) \mid d s \\
\quad+|\lambda| \sum_{j=1}^{N} \int_{x_{j-1}}^{x_{j}}\left(\xi-x_{j-1}\right)\left|\frac{\partial}{\partial \xi}\left[K\left(x_{i}, \xi\right) u(\xi)\right]\right| d \xi \\
\leq \chi_{i}^{-1} h^{-1}|\lambda| \int_{x_{i-1}}^{x_{i+1}} d x \varphi_{i}(x) \int_{0}^{l}\left(x-x_{i}\right)\left|\frac{\partial}{\partial \xi} K(\xi, s) u(s)\right| d s \\
+|\lambda| h \int_{0}^{l}\left|\frac{\partial}{\partial \xi}\left[K\left(x_{i}, \xi\right) u(\xi)\right]\right| d \xi \\
\leq C h|\lambda|+|\lambda| h \int_{0}^{l}\left\{\left|\frac{\partial K\left(x_{i}, \xi\right)}{\partial \xi}\right||u(\xi)|+\left|K\left(x_{i}, \xi\right)\right|\left|u^{\prime}(\xi)\right|\right\} d \xi \\
\leq C\left\{h+h \int_{0}^{l}\left(1+\frac{1}{\sqrt{\varepsilon}}\left(e^{-\frac{\sqrt{\alpha} \xi}{\sqrt{\varepsilon}}}+e^{-\frac{\sqrt{\alpha}(l-\xi)}{\sqrt{\varepsilon}}}\right)\right) d \xi\right\} \leq C h .
\end{aligned}
$$

Thus from (4.3)-(4.5) we see easily the estimate

$$
\|R\|_{\infty} \leq C h .
$$

The bound (4.6) together with (4.2) completes the proof. 


\section{NUMERiCAL RESUlts}

Consider the particular problem with

$$
\begin{aligned}
& a(x)=1, \quad K(x, s)=x, \quad f(x)=x-\varepsilon+\varepsilon e^{-\frac{x}{\varepsilon}}, \quad x \in(0,1), \\
& \lambda=\frac{1}{2}, \quad A=1, \quad B=2-\varepsilon+\varepsilon e^{-\frac{1}{\varepsilon}},
\end{aligned}
$$

The exact solution is given by

$$
u(x)=\frac{e^{-\frac{x}{\sqrt{\varepsilon}}}+e^{\frac{x-1}{\sqrt{\varepsilon}}}-e^{\frac{x-2}{\sqrt{\varepsilon}}}-e^{-\frac{x+1}{\sqrt{\varepsilon}}}}{1-e^{-\frac{2}{\sqrt{\varepsilon}}}}+x-\varepsilon+\varepsilon e^{-\frac{x}{\sqrt{\varepsilon}}} .
$$

We define the exact error $e_{\varepsilon}^{h}$ and the computed $\varepsilon$-uniform maximum pointwise error $e^{h}$ as follows:

$$
e_{\varepsilon}^{h}=\|y-u\|_{\infty}, \quad \quad e^{h}=\max _{\varepsilon} e_{\varepsilon}^{h} .
$$

We also define the computed parameter-uniform rate of convergence to be

$$
p^{h}=\ln \left(e^{h} / e^{h / 2}\right) / \ln 2 .
$$

The resulting errors $e^{h}$ and the corresponding numbers $p^{h}$ for various values $\varepsilon$ and $h$ are listed in Table 1.

Table 1 Exact errors $e_{\varepsilon}^{h}$, computed $\varepsilon$-uniform errors $e^{h}$ and convergence rates $p^{h}$ on $\omega_{N}$.

\begin{tabular}{lllllll}
\hline$\varepsilon$ & $h=1 / 32$ & $h=1 / 64$ & $h=1 / 128$ & $h=1 / 256$ & $h=1 / 512$ & $h=1 / 1024$ \\
\hline 1 & 0.00343868 & 0.00198874 & 0.00110332 & 0.00060368 & 0.00030394 & 0.00015197 \\
& 0.79 & 0.85 & 0.87 & 0.99 & 1.00 & \\
$2^{-4}$ & 0.01032126 & 0.00605257 & 0.00338123 & 0.00185003 & 0.00094445 & 0.00047551 \\
& 0.77 & 0.84 & 0.87 & 0.97 & 0.99 & \\
$2^{-8}$ & 0.01125894 & 0.00660244 & 0.00368841 & 0.0020181 & 0.00103025 & 0.00051871 \\
& 0.77 & 0.84 & 0.87 & 0.97 & 0.99 & \\
$2^{-12}$ & 0.011200979 & 0.00656845 & 0.00366942 & 0.00200771 & 0.00102495 & 0.00051604 \\
& 0.77 & 0.84 & 0.87 & 0.97 & 0.99 & \\
$2^{-16}$ & 0.0112049 & 0.00657075 & 0.00367071 & 0.00200842 & 0.00102531 & 0.00051622 \\
& 0.77 & 0.84 & 0.87 & 0.97 & 0.99 & \\
\hline$e^{h}$ & 0.01125894 & 0.00660244 & 0.00368841 & 0.0020181 & 0.00103025 & 0.00051622 \\
$p^{h}$ & 0.77 & 0.84 & 0.87 & 0.97 & 0.99 & \\
\hline
\end{tabular}

The obtained results show that the convergence rate of difference scheme is essentially in accord with the theoretical analysis. 


\section{CONCLUSiON}

A boundary-value problem for a second order singularly perturbed Fredholm integro-differential equation has been considered. For the numerical solution of this problem, we proposed a fitted finite difference scheme on a uniform type mesh. The difference scheme is constructed by the method of integral identities with the use of exponential basis functions and interpolating quadrature rules with the weight and remainder terms in integral form. It is shown that the method displays uniform convergence independently of the perturbation parameter in the discrete maximum norm. We have implemented the present method on a example. Numerical results were carried out to show the efficiency and accuracy of the method.

\section{REFERENCES}

[1] G. M. Amiraliyev and Y. D. Mamedov, "Difference schemes on the uniform mesh for singularly perturbed pseudo-parabolic equations." Turkish J. Math., vol. 19, pp. 207-222, 1995.

[2] G. M. Amiraliyev and Ö. Yapman, "On the Volterra delay-integro-differential equation with layer behavior and its numerical solution." Miskolc Math. Notes, vol. 20, no. 1, pp. 75-87, 2019, doi: 10.18514/mmn.2019.2424.

[3] G. M. Amiraliyev and B. Yilmaz, "Fitted difference method for a singularly perturbed initial value problem." Int. J. Math. Comput., vol. 22, no. 1, pp. 1-10, 2014.

[4] R. Bellman and K. L. Cooke, Differential difference equations., New York, 1963.

[5] M. C. Bonis and C. Laurita, "Numerical treatment of second kind Fredholm integral equations systems on bounded intervals." J. Comput. Appl. Math., vol. 217, no. 1, pp. 64-87, 2008, doi: 10.1016/j.cam.2007.06.014.

[6] M. J. Emamzadeh and M. T. Kajani, "Nonlinear Fredholm integral equation of the second kind with quadrature methods." Journal of Mathematical Extension, vol. 4, no. 2, pp. 51-58, 2010.

[7] M. Feckan, "Singularly perturbed higher order boundary value problems." J. Differential Equations, vol. 111, pp. 79-102, 1994, doi: 10.1006/jdeq.1994.1076.

[8] O. A. Gegele, O. P. Evans, and D. Akoh, "Numerical solution of higher order linear Fredholmintegro-differential equations." American Journal of Engineering Research, vol. 3, no. 8, pp. 243 247, 2014.

[9] Z. Jackiewicz, M. Rahman, and B. D. Welfert, "Numerical solution of a Fredholm integrodifferential equation modelling $\theta$ neural networks." Appl. Math. Comput., vol. 195, no. 2, pp. 523-536, 2008, doi: 10.1016/j.amc.2007.05.031.

[10] M. F. Karim, M. Mohamad, M. S. Rusiman, N. Che-him, R. Roslan, and K. Khalid, "ADM for solving linear second-order Fredholm integro-differential equations." Journal of Physics: Conference Series, vol. 995, p. 012009, 2018, doi: 10.1088/1742-6596/995/1/012009.

[11] S. Karimi and M. Jozi, "Numerical solution of the system of linear Fredholm integral equations based on degenerating kernels." J. Pure Appl. Math., vol. 6, no. 1, pp. 111-119, 2015.

[12] M. Kudu, I. Amirali, and G. M. Amiraliyev, "A finite-difference method for a singularly perturbed delay integro-differential equation.” J. Comput. Appl. Math., vol. 308, pp. 379-390, 2016, doi: 10.1016/j.cam.2016.06.018.

[13] N. Kurt and M. Sezer, "Polynomial solution of high-order Fredholm integro-differential equations with constant coeffcients." J. Franklin Inst., vol. 345, no. 8, pp. 839-850, 2008, doi: 10.1016/j.jfranklin.2008.04.016.

[14] P. Kythe and P. Puri, Computational methods for linear integral equations. Boston: Birkhäuser Basel, 2002. doi: 10.1007/978-1-4612-0101-4. 
[15] J. J. H. Miller, E. O’Riordan, and G. I. Shishkin, Fitted Numerical methods for singular perturbation problems. Singapore: World Scientific, 1996.

[16] A. H. Nayfeh, Introduction to perturbation techniques. New York: Wiley, 1993.

[17] R. E. O'Malley, Singular perturbation methods for ordinary differential equations. New York: Springer-Verlag, 1991.

[18] A. D. Polyanin and A. V. Manzhirov, Handbook of integral equations. Boca Raton, Fla, USA: Chapman and Hall/CRC, 2008.

[19] H. G. Roos, M. Stynes, and L. Tobiska, Numerical methods for singularly perturbed differential equations. Berlin: Springer-Verlag, 1996.

[20] Q. Xue, J. Niu, D. Yu, and C. Ran, “An improved reproducing kernel method for Fredholm integrodifferential type two-point boundary value problems." Int. J. Comput. Math., vol. 95, no. 5, pp. 1015-1023, 2018, doi: 10.1080/00207160.2017.1322201.

Authors' addresses

Gabil M. Amiraliyev

Erzincan Binali Yıldırım University, Faculty of Arts and Sciences, Department of Mathematics, 24100, Erzincan, Turkey

E-mail address: gabilamirali@yahoo.com

\section{Muhammet Enes Durmaz}

Kırklareli University, Department of Information Technology, 39000, Kırklareli, Turkey

E-mail address: menesdurmaz025@gmail.com

\section{Mustafa Kudu}

Erzincan Binali Yıldırım University, Faculty of Arts and Sciences, Department of Mathematics, 24100, Erzincan, Turkey

E-mail address: muskud28@yahoo.com 